\title{
Application of EMD in Signal Processing of Track State Detection
}

\author{
Zhu Wenfa' Ma Huizhen ${ }^{2}$ \\ ${ }^{1}$ hanghai University of Engineering Science College of Urban Rail Transportation \\ Shanghai, China \\ ${ }^{2}$ hanghai University of Engineering Science College of Library \\ Shanghai, China
}

\begin{abstract}
Key words: Track state; Noise; Filtering; EMD; Continuous mean square error criterion
Abstract: In this paper, a filtering method based on empirical mode decomposition (EMD) is proposed for the accuracy of measurement, which is regularly influenced by the noise in the output signal of inertial measurement unit in the track state detecting process, the multi-scale filtering characteristics are analyzed at the same time. The Cut-off point $\mathrm{K}$ is determined between the modes dominated by noise and signal in the IMF component using continuous mean square error criterion, so as to realize the purpose of filtering. The algorithm performance is verified through the simulation signal. The validation based on the collected railway line acceleration data shows that the noise in railway line state acceleration detection signals can be effectively eliminated through the EMD de-noising algorithm method using continuous mean square error criterion.
\end{abstract}

\section{Introduction}

Engineering signal filter processing is always a research hotspot in the field of signal processing. In engineering, the signals collected from scene are inevitable to be disturbed by noise which is bound to influence the accuracy of the final results even leads to mistakes. So it is of great significance to make a research on how to de-noise the collected signals in order to enhance the accuracy of processing. The traditional filtering methods are Fourier transform, Wiener filtering method and Wavelet transform et al.[1]Filtering method based on EMD is a brand new signal processing theory. It is a space-time filter whose measurement is according to local characteristic time scale of the signal. This method not only has the same multi-resolution feature as wavelet transform but also solves the problem of wavelet basis selection in wavelet transform. [2]In the track state detection, strap-down inertial technology [3] whose inertial measurement unit can output the signals of acceleration and angular velocity. Finally, these signals form a mathematics analytic platform. However, the signals output by inertial measurement unit and collected from the scene are digital signals which are transformed by internal signal module of inertial measurement unit [4]. These signals are usually exist certain DC offset and contain some noise. Therefore, the effect of de-noise processing of acceleration velocity will directly affect the precision of the integral and then influence the accuracy of the whole system.

\section{Empirical Mode Decomposition (EMD)}

In 1998, NordenE.Huang et al proposed a non-linear and non-stationary signal processing method 
which is called Hilbert-Huang Transform (HHT). It can be divided into three parts [5]:

(1) According to real fluctuation size of signals, we use EMD to decompose the signals step by step which is also called signal stabilization processing. Then we can get a series of Intrinsic Mode Function (IMF) of different characteristic scale.

(2) Carrying out Hilbert transform on every decomposed Intrinsic Mode Function to obtain the corresponding Hilbert spectrum.

(3) Combining all the Hilbert spectrum of IMF to obtain Hilbert spectrum of every time which can reflect the frequency value, amplitude value of the original signals.

\section{Filtering Method Based on EMD}

\section{Multi-scale filtering characteristic of EMD}

We can get well-ordered and multi-stage IMF from high frequency to low frequency after the signals are decomposed by EMD. Substantially, this is a process of high frequency filtering to low frequency filtering which embodies self-adaption filtering characteristic of EMD. According to filtering characteristic of EMD, Flandrin proposed the construction method of filter bank [8] which is shown below.

(1) High-pass filter: Using the former several IMF components of higher frequency to reconfigure the original signals under the condition of getting rid of the remaining IMF components of lower frequency.

(2) Low-pass filter: Using the latter several IMF components of lower frequency to reconfigure the original signals under the condition of getting rid of the remaining IMF components of higher frequency.

(3) Band-pass filter : Getting rid of the latter several IMF components of lower frequency and the former several IMF components of higher frequency at the same time.

Obviously, there exists some differences between this filter bank and the traditional filter of fixed cutoff frequency. The pass-band cutoff frequency of this filter bank changes with the input signals which makes it self-adaptive.

\section{EMD Filtering Based on Continuous Mean Square Error Criterion}

Assuming $y(t)$ signifying the signals polluted by noise, $x(t)$ signifying the original signals, $\mathrm{n}(\mathrm{t})$ signifying white Gaussian noise, namely: $y(t)=x(t)+n(t)$

After $y(t)$ is decomposed by EMD, we can obtain finite IMF components that are ranked from high frequency to low frequency. The IMF of small order correspond to signals of high frequency which are also sharp parts or noise of signals. The IMF of big order correspond to signals of low frequency which are thought to be less effected by noise. For any IMF components, either the signals are dominant mode or the noise is dominant mode. Besides, there must be a demarcation point $\mathrm{K}$, before which IMF components take the noise as the dominant mode, after which IMF components take the signals as the dominant mode. Therefore, the key assignment of de-noising based on EMD filtering is to find out the demarcation point $\mathrm{K}$. Boudraa [9] proposed a method to search the demarcation point $\mathrm{K}$ using continuous mean square error criterion. This method aims to find out the K-th IMF component whose noise energy distribution changes abruptly corresponding to formula 2. Then the IMF components after K are reconstructed as shown in formula 4.

$$
\begin{aligned}
& \operatorname{CMSE}\left(\mathrm{x}_{\mathrm{k}}^{r}, \mathrm{x}_{\mathrm{k}+1}^{g}\right)=\frac{1}{N} \sum_{\mathrm{i}=1}^{N}\left[\mathrm{x}_{\mathrm{k}}^{g}\left(\mathrm{t}_{\mathrm{i}}\right)-\right. \\
& \left.\mathrm{x}_{\mathrm{k}+1}^{g}\left(\mathrm{t}_{\mathrm{i}}\right)\right]^{2}=\frac{1}{N} \sum_{\mathrm{i}=1}^{N}\left[\mathrm{IMF}_{\mathrm{k}}\left(\mathrm{t}_{\mathrm{i}}\right)\right]^{2}(\mathrm{k}=1, \ldots, \mathrm{n}-
\end{aligned}
$$


Definition of demarcation point $\mathrm{K}: \mathrm{k}=$

$\operatorname{argmin}_{1 \leq k \mathrm{~N}}\left[\mathrm{CMSE}\left(x_{\mathrm{k},}^{\prime} x_{\mathrm{k}+1}^{\prime}\right)\right]+1$

$\mathrm{x}^{\prime}(\mathrm{t})=\sum_{\mathrm{i}=\mathrm{k}+1}^{\mathbb{N}} \mathrm{imf}_{\mathrm{i}}(\mathrm{t})$

\section{De-noising performance analysis}

In the detection of rail state, there exist the effect of test circuit noise and external environment in the course of transforming the sensor signals collected from scene into digital signals. The acceleration velocity signals observed in the computer are consisted of periodic component, constant component and noise. So we can use the below formula to describe the acceleration velocity signals.

$$
f(t)=A \sin \left(\omega_{0} t+\varphi\right)+\mu+\sigma(t), t=0,1,2,3, \ldots, n-1
$$

A-Amplitude value; $\varphi$-Initial phase; $\omega_{0}-$ Fundamental frequency; $\mu-$ DC value ; $\sigma(t)-W h i t e$ Gaussian noise

We can eliminate DC value through mean filtering. We pass over the introduction here. We will use simulation experiment to evaluate the EMD de-noising performance based on continuous mean square error criterion.

Using EMD de-noising method based on continuous mean square error criterion to de-noise $\mathrm{y}^{(\mathrm{t})}$ which is shown in Figure 1.y $(\theta)$ derives from superposing $x(t)=2 \sin \left(20 \pi t+\frac{\pi}{4}\right)$ on white Gaussian noise.

Energy curve of IMF components is shown in Figure 2 after $y(t)$ is decomposed by EMD.

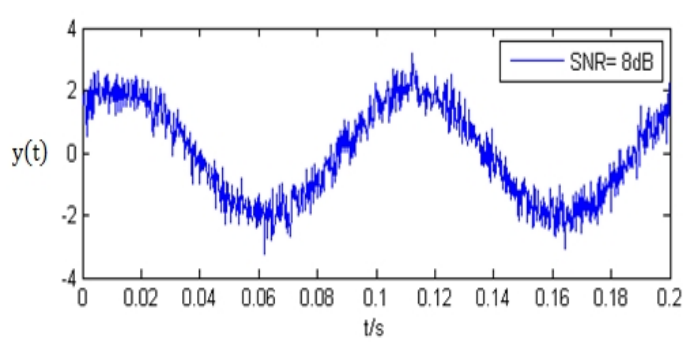

Figure 1 Oscillogram of $y(t)$

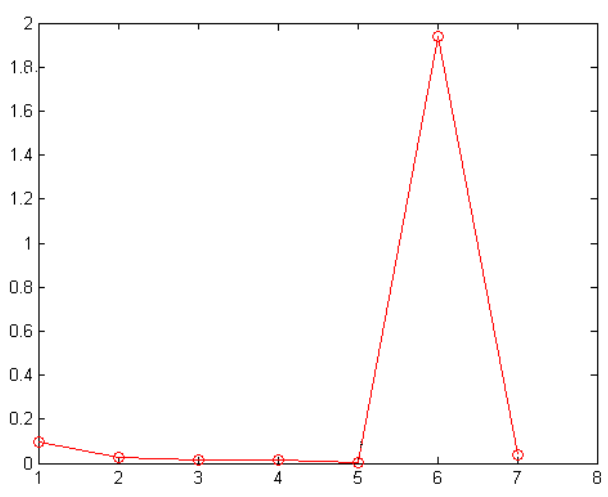

Figure 2 Energy change curve

According to Figure 2, the demarcation point $\mathrm{K}$ of noise dominant mode and signal dominant mode for $y(t)$ is five. Therefore, we can get the de-noising result $x^{\prime}(\oplus$ (Figure 3$)$ through filtering the IMF components of the former five noise dominant mode and reconstructing the IMF components of the sixth and the seventh signal dominant mode. As you can see in Figure 3, the de-noised signals are consistent with the real signals which has a better de-noising effect. 


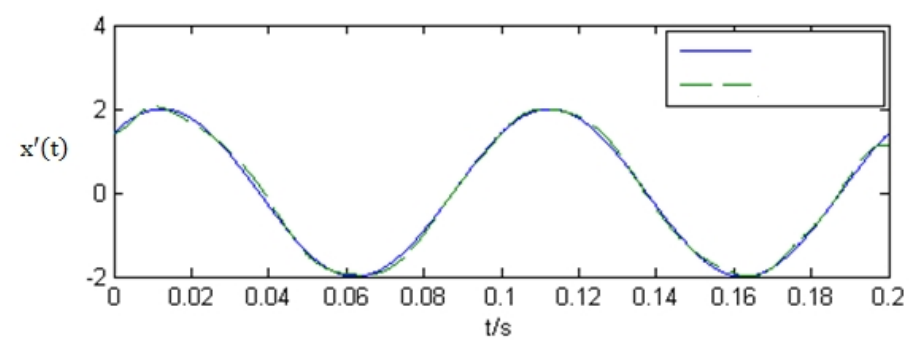

Figure 3 De-noising result

\section{Experiment analysis}

In this experiment, we let testing car loaded with inertial measurement unit pass an analog line section in order to collect the acceleration velocity signals of the moving car along the $\mathrm{x}$-axis, $\mathrm{y}$-axis and z-axis direction which are shown in Figure 4.

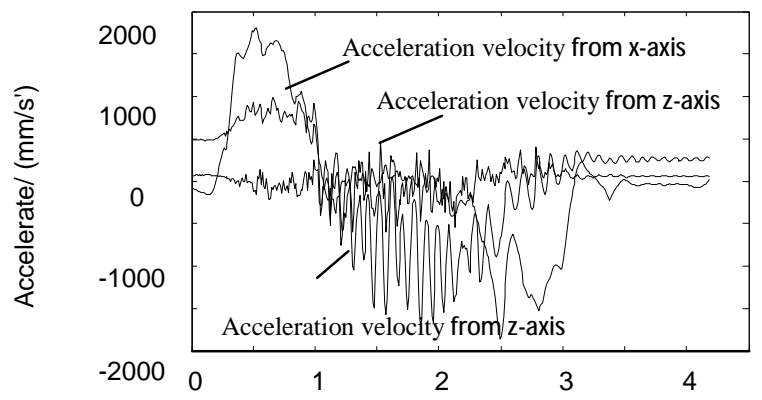

Figure 4 Acceleration velocity curve

Firstly, we use the mean filtering method to eliminate the DC value involved in acceleration velocity signals. Then, we utilize EMD de-noising method based on continuous mean square error criterion to de-noise the acceleration velocity signals whose DC value is eliminated [10]. We conduct EMD decomposition on acceleration velocity signals. We search the demarcation point $\mathrm{K}$ between the noise dominant mode and signal dominant mode in IMF components through continuous mean square error criterion. As you can see in Figure 5 to 6 , the demarcation point $\mathrm{K}$ along the $\mathrm{x}$-axis, $\mathrm{y}$-axis and $\mathrm{z}$-axis direction is six, three and three respectively.

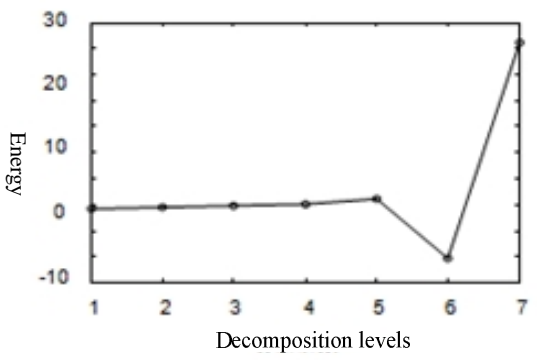

Figure 5 Energy change curve of acceleration velocity on $\mathrm{x}$-axis 


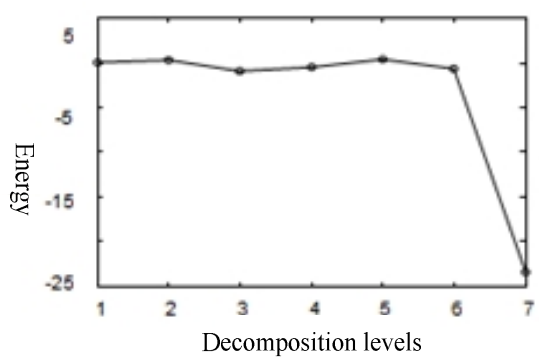

Figure 6 Energy change curve of

acceleration velocity on y-axis

\section{Results}

This paper uses EMD de-noising method based on continuous mean square error criterion to de-noise the acceleration velocity signals output by inertial measurement unit. Then we conduct integral computing on the de-noised acceleration velocity signal. We are able to get the motion trail of the little car using the attitude matrix to transform the motion information in carrier coordinate system into the motion information in geographic coordinate system. The experimental results show that the EMD de-noising method based on continuous mean square error criterion can de-noise the noise of signals effectively and satisfy the detection precision requirement better.

\section{Acknowledgment}

The project is jointly supported by the National Natural Science Foundation of China (51478258), The Training Subsidy Scheme Of Young Teachers in Shanghai (ZZGJD13072), and the Shanghai Graduate Education Innovation Project in Layout and Construction Project (13sc002).

\section{References}

[1] Hu Ying,Yang Jie,Zhou Yue. Fractal noise filtering based on multi-scale Wiener filter [J]. Journal of electronics, 2003, 04:560-563P.

[2] Sun Jinbao, Zhu Yongli, Liu Liqing et al. De-noise research of insulator leakage current based on EMD [J]. Journal of North China Electric Power University, 2010, 37(6):1-5, 22.

[3] Zhu Wenfa,Chai Xiaodong,Zheng Shubin et al. Design of signal acquisition and processing system of inertial measurement unit based on LabVIEW[J].Computer Measurement \& Control.2012, 20(6):1697-1698,1706.

[4] Zheng Shubin, Lin Jianhui, Lin Guobin. The detection and realization of track irregularity of magnetic levitation based on inertial method [J].Journal of electronic measurement and instrument, 2007, 21(1): 61-65.

[5] Li Lin. Research and application of HHT time-frequency analysis method [D].Jilin University, 2006, 30-35.

[6] N.E.Huang,ShenZ,S.R.Long,etal.The empirical mode decomposition and the Hilbert spectrum for nonlinear and non-stationary time series analysis, ProcRsoc Lond.1998, 454:56-78.

[7] Abdel OuahabBoudraa, Jean-Christophe Cexus . EMD-Based Signal Filtering . IEEE Transactions on Instrumentation and Measurement.2007,56(6): 2196-2202.

[8] P.Flandrin. Empirical mode decomposition as a filter bank. IEEE Signal Processing Leuels, 2003,11(2):112-114P.

[9] Chen Ping,Li Qingmin,Zhao Tong. Review on research progress of instantaneous frequency estimation algorithm[J]. Electron Measurement and Instrument,2006,07:1-7. 
[10] Liu Xinguang,Chai Xiaodong,Zheng Shubin et al. De-noising processing of acceleration signal in inertial measurement unit[J].Instrument Technique and Sensor.2013,10:97-99 